\title{
Modulation of Nrf2/ARE Pathway by Food Polyphenols: A Nutritional Neuroprotective Strategy for Cognitive and Neurodegenerative Disorders
}

\author{
Giovanni Scapagnini, \\ Department of Health Sciences, University of Molise, Campobasso, Italy \\ Vasto Sonya, \\ Immunosenescence Unit, Department of Pathobiology and Biomedical Methodologies, University \\ of Palermo, Palermo, Italy
}

\begin{abstract}
Abraham G. Nader, Department of Physiology and Pharmacology, College of Medicine, University of Toledo, Toledo, $\mathrm{OH}$, USA
\end{abstract}

\section{Caruso Calogero,} Immunosenescence Unit, Department of Pathobiology and Biomedical Methodologies, University of Palermo, Palermo, Italy

Davide Zella, and Department of Biochemistry and Molecular Biology, Institute of Human Virology-School of Medicine, University of Maryland, Baltimore, MD, USA

\section{Galvano Fabio}

Department of Biological Chemistry, Medical Chemistry and Molecular Biology, University of atania, Catania, Italy

\section{Abstract}

In recent years, there has been a growing interest, supported by a large number of experimental and epidemi-ological studies, for the beneficial effects of some phenolic substances, contained in commonly used spices and herbs, in preventing various age-related pathologic conditions, ranging from cancer to neurodegenerative diseases. Although the exact mechanisms by which polyphenols promote these effects remain to be elucidated, several reports have shown their ability to stimulate a general xenobiotic response in the target cells, activating multiple defense genes. Data from our and other laboratories have previously demonstrated that curcumin, the yellow pigment of curry, strongly induces heme-oxygenase-1 (HO-1) expression and activity in different brain cells via the activation of heterodimers of NF-E2-related factors 2 (Nrf2)/antioxidant responsive element (ARE) pathway. Many studies clearly demonstrate that activation ofNrf2 target genes, and particularly HO-1, in astrocytes and neurons is strongly protective against inflammation, oxidative damage, and cell death. In the central nervous system, the HO system has been reported to be very active, and its modulation seems to play a crucial role in the pathogenesis of 
neurodegenerative disorders. Recent and unpublished data from our group revealed that low concentrations of epigallocatechin-3-gallate, the major green tea catechin, induces HO-1 by ARE/ Nrf2 pathway in hippocampal neurons, and by this induction, it is able to protect neurons against different models of oxidative damages. Furthermore, we have demonstrated that other phenolics, such as caffeic acid phenethyl ester and ethyl ferulate, are also able to protect neurons via HO-1 induction. These studies identify a novel class of compounds that could be used for therapeutic purposes as preventive agents against cognitive decline.

\section{Keywords}

Heterodimers of NF-E2-related factors 2 (Nrf2); Antioxidant responsive element (ARE); Heme oxygenase 1 (HO-1); Neurodegenerative disorders; Alzheimer's disease; Polyphenols; Curcumin; (-)- epigallocatechin-3- gallate (EGCG); Brain ageing

\section{Introduction}

Alzheimer's disease (AD) is the most common form of dementia and the fifth leading cause of death in Americans aged 65 years and older, but whereas other major causes of death have been decreasing, deaths attributable to $\mathrm{AD}$ have been rising dramatically in the last decade (47\% increase between 2000 and 2006) [1]. There is abundant evidence that both oxidative stress and inflammatory mechanisms within the CNS contribute to cognitive impairment as AD. Neurodegeneration in AD appears to be multifactorial, whereby several biochemical processes operate sequentially and/or in parallel $[2,3]$.

Oxidative stress has been implicated in a variety of pathophysiological conditions, including neurodegenerative, and is one of the earliest pathological changes in AD. In particular, cortical and hippocampal oxidative stress is a very early event in the pathogenesis of sporadic AD and correlates with the development of specific cognitive deficits in this condition. Free radicals are produced from a number of sources, among which are enzymatic, mitochondrial, and redox metal ion-derived sources [4].

Oxidative damage to key intracellular targets such as DNA or proteins by free radicals has been shown to be a major cause of the neuronal cell death related to AD. For example, recent studies have applied redox proteomic, a branch of proteomics that identifies oxidatively modified proteins, to characterize specific proteins in brain aging, and a number of proteins that are oxidatively modified in AD have been identified [5]. Aging, the major risk factor for $\mathrm{AD}$, leads to loss of free radical scavenging ability by endogenous mechanisms, and it is widely held that free radical-induced oxidative stress increases in brain aging [6]. Activation of antioxidant pathways is particularly important for tissues with relatively weak antioxidant defenses, such as the brain. Increasing evidence points to the notion that reduced cellular expression and activity of antioxidant proteins and the consequent oxidative stress are fundamental causes for brain aging processes and neurodegenerative diseases [7]. In fact, compared to other organs, the brain is more susceptible to oxidative stress for the following reasons: (a) its high content of peroxidizable unsaturated fatty acids; (b) high oxygen consumption per unit weight; (c) high content of lipid peroxidation key ingredients (iron and ascorbate); and (d) the scarcity of antioxidant 
defense systems. On the other hand, irrespective of the source and mechanisms that lead to the generation of intracellular toxic oxidants, mammalian cells have developed highly refined inducible systems to counteract stressful conditions [8].

When properly activated, each one of these cell systems has the possibility to restore cellular homeostasis and resume the ability to fight off oxidation. Thus, the malfunctioning of cellular stress response machinery has been indicated as one of the major causes of inflammatory and oxidative states of aging.

Since oxidative stress may underlie some aspects of AD neurodegeneration, and since to date, most of the available treatments are merely symptomatic [9], considerable research has been aimed at reducing the effects of oxidative stress in order to prevent AD progress, by use of free radical scavengers [10]. Thus, one therapeutic strategy is to delay the onset of $\mathrm{AD}$ dementia sufficiently long as to slow the neuronal damage associated with $A \beta$-induced oxidative stress, particularly $\mathrm{A} \beta$-induced lipid peroxidation. Brain-accessible antioxidants potentially may provide the means of implementing this therapeutic strategy of delaying the onset of $\mathrm{AD}$, acting as neuroprotective agents. By definition, neuroprotection is an effect that may result in salvage, recovery, or regeneration of the nervous system, its cells, structure, and function. Although there are several lines of evidence supporting the hypothesis that neuroprotection may be a practical and achievable pharmacological target, few effective compounds have been developed for clinical application. To date, the use of antiapoptotic therapies for neurodegenerative disorders has not been successful, particularly because many of the compounds have high levels of toxicity. Moreover, interfering with apoptotic pathways has often resulted in an augmented risk of cancerogenesis. On the other hand, the use of safe antioxidant compounds, such as $\boldsymbol{a}$-tocopherol, in the treatment of neurodegenerative disorders is strongly limited by the difficulty to reach an active concentration of these molecules in the brain [11]. Thus, in the field of the pharmacological treatment of neurodegenerative disorders, there remain the need and desire for safe, nontoxic, and orally effective pharmacological agents able to interfere with oxidative and inflammatory processes.

\section{Nrf2, HO-1, and Neuroprotection}

Although the mechanisms that lead to oxidative stress and inflammation in the brain are various (still poorly understood), a number of studies suggest an important role for the transcription factor nuclear factor E2-related factor 2 (Nrf2)-mediated signaling in neuroprotection $[12,13]$. Nrf2, a member of the Cap ' $n$ 'Collar family of transcription factors, is sequestered in the cytoplasm by binding to protein Kelch ECH associating protein 1 (Keap1) in non-stimulated conditions. Keap1 plays a central role in regulation of the Nrf2 response. Under normal conditions, Nrf2 is targeted by Keap1, which promotes Nrf2 proteasomal degradation via interactions with an ubiquitin ligase [14]. Keap1 further functions as a sensor of stress signals, through stress-induced oxidation of key cysteine residues that lead to conformational changes and the inability to bind Nrf2 [15]. Several stimuli, including oxidative stress and electrophiles, lead to the disruption of Nrf2/Keap1 complex, freeing Nrf2 for translocation to the nucleus where it interacts with basic leucine zipper transcription factors such as Maf and Jun family members and binds to a cis-acting 
element, the antioxidant response element (ARE, also called EpRE or OSRE). Nrf2-ARE is a major pathway regulating phase II antioxidant responses, triggering the simultaneous expression of numerous protective enzymes and scavengers. Recently, a genome-scale analysis of the regulatory network governed by Nrf2 has been performed, using a combination of high-throughput sequencing for ChIP and microarraybased gene-expression profiling [16]. The authors have identified 645 basal and 654 inducible direct target genes of Nrf2, with 244 genes at the intersection, all involved in stress response and cell proliferation. The ARE is found in several cytoprotective inducible genes related to the so-called cellular stress response, such as the gluthatione- $S$-transferases, heme oxygenase 1 (HO-1), glutamate cysteine ligase,ferritin, gamma glutamyl cysteine synthetase, $\mathrm{NAD}(\mathrm{P}) \mathrm{H}$ quinone oxidoreductase thioredoxin, and peri-redoxins, and this emphasizes Nrf2 as a central node in cell survival response.

It has been shown that in neurodegenerative diseases, $\mathrm{Nrf} 2$ expression is altered in both neurons and astrocytes. In a Parkinson model, Nrf2 deficiency increased sensitivity to MPTP or 6-hydroxydopamine administration in mice whereas overexpression of Nrf2 in astrocytes abolished MPTP or 6-hydroxydopamine toxicity [17]. Furthermore, Nrf2-knockout mice showed exacerbated gliosis and dopaminergic nigrostriatal degeneration [18] and stronger activation of microglia when treated with MPTP compared to wild type, highlighting the importance of Nrf2 pathway in the control of microglial function in Parkinson's disease progression [19]. The ability of Nrf2 overexpression to protect motor neurons, in a transgenic mouse model of amyotrophic lateral sclerosis (ALS), has been associated to an increased glutathione secretion from astrocytes [20]. Also, breeding a mouse with Nrf2overexpressing astrocytes with the G93A SOD model of ALS produced delayed onset of symptoms and improved survival [21]. The potential role of the Nrf2-ARE pathway in AD genesis and progression is supported by the fact that Nrf2 levels are reduced in the hippocampus of $\mathrm{AD}$ patients [22]. Moreover, in transgenic $\mathrm{AD}$ mice expressing mutated human amyloid precursor protein (APP) and presenilin 1 (PS1) genes (APP/ PS1 mice), the Nrf2-ARE pathway is attenuated at the time of $A \beta$ deposition [23]. Importantly, Nrf2 overexpression in vitro protects against neurotoxicity of $A \beta$ and is associated with increased expression of Nrf2 target genes and reduced oxidative stress [23, 24]. A recent study demonstrated that direct intrahippocampal gene delivery of Nrf2, by a lentiviral vector, results in a reduction in spatial learning deficits in aged APP/PS1 mice [25]. Although it has been proposed that the beneficial effect of Nrf2 induction in the central nervous system is based on the actions of astrocytes [26], this study showed that a beneficial behavioral outcome in APP/PS1 mice can be achieved by modulating levels of Nrf2 specifically in neurons.

Among the genes activated by Nrf2/ARE, HO-1 has been the object of intensive studies in the brain for its potential role in protecting neurons against cell death. HO-1 is a ubiquitous and redox-sensitive inducible stress protein and, together with the constitutive isozyme HO-2, provides the first and rate-limiting step in heme degradation [27]. HO cleaves the heme ring via oxidation at the alpha methene bridge to give biliverdin, gaseous carbon monoxide, and free iron. Biliverdin is subsequently converted to bilirubin by biliverdin reductase, and both these molecules can act as intracellular antioxidants. A substantial body of evidence demonstrates that both bilirubin and carbon monoxide (CO) effectively 
contribute to modulate important physiological processes within the cardiovascular, immune, and nervous systems. These include the regulation of vessel tone, inhibition of platelet aggregation, and prevention of cell death and tissue injury. In the CNS, the HO system has been reported to be very active, and its modulation seems to play a crucial role in the pathogenesis of neurodegenerative disorders. Deregulation of the HO system has been associated with the pathogenesis of AD [28], multiple sclerosis, and brain aging [29, 30].

The HO-1 gene has ARE consensus sequence, responding to Nrf2, as well as heat shock, $\mathrm{AP} 1, \mathrm{AP} 2$, and NF- $\mathrm{\kappa B}$ binding sites in its promoter region, and it is rapidly upregulated by oxidative and nitrosative stresses as well as by many toxic compounds. Many studies clearly demonstrate that activation of HO-1 in neurons is strongly protective against oxidative damage and cell death [31, 32]. In fact, the activation of HO-1 seems to represent an important defensive mechanism for neurons exposed to oxidative stress.

More recently, HO-1 has also been recognized to exhibit important immunomodulatory and anti-inflammatory functions. A potential link between HO-1 and inflammation has initially been shown in an animal model, in which specific upregulation of HO enzyme activity attenuated complement-dependent inflammation [33]. Shortly thereafter, it has been demonstrated in a HO-1 knockout mouse model that these animals develop a chronic inflammatory disease and are highly susceptible to an experimental sepsis induced by the classical pro-inflammatory mediator endotoxin [34]. Importantly, phenotypical alterations in the only known human case of genetic HO-1 deficiency are similar to those observed in HO-1 knockout mice [35]. Independently, targeted over-expression of HO-1 has been shown to have beneficial effects in various experimental animal models of inflammation [36]. Pharmacological induction of HO-1 and exposure to the end-product of HO-1 activity, CO, prevents the development of experimental cerebral malaria, by inhibiting blood-brain barrier (BBB) disruption, brain microvasculature congestion, and neuroinflammation [37]. Induction of experimental autoimmune encephalomyelitis (EAE), a model of multiple sclerosis, in HO-1-knockout mice led to enhanced CNS demyelination, paralysis, and mortality, as compared with wild-type mice [38]. In the same study, induction of HO-1 using cobalt protoporphyrin IX, a specific HO-1 inducer, modulated ongoing autoimmune neuroinflammation, thereby reverting paralysis and leading to disease remission in mice with previously established EAE.

On the base of such evidences, modulation of Nrf2 and HO-1 in the brain has been recently suggested as a potential pharmaceutical strategy for the treatment and prevention of neurodegenerative disorders and pathologic brain aging. Synthetic triterpenoids, particularly those which are analogs of 2-Cyano-3,12-Dioxooleana-1,9-Dien-28-Oic acid (CDDO), have been shown to induce Nrf2/ARE pathway in both cell culture and in mice. In an AD transgenic mouse model, CDDO-methyl amide reduced protein carbonyl levels, a biomarker of protein oxidation, improved memory, and reduced amyloid pathology [39]. A recent study has demonstrated that two CDDO analogs were able to activate the Nrf2/ARE pathway in the N171-82Q transgenic mouse model of Huntington's disease. Nrf2/ARE activation resulted in the upregulation of different cytoprotective genes, such as HO-1, decreased oxidative stress, improved motor performance, and increased survival and rescue of striatal atrophy in the mice's brain. Plant-derived pesticide plumbagin has been recently identified as 
a novel potent activator of the Nrf2/ARE pathway, and its administration has been shown to significantly reduce brain damages and neurological deficits in a mouse model of focal ischemic stroke [40]. Several nutritional factors have also been demonstrated to interfere with Nrf2 signaling in the brain. A recent study demonstrates for the first time that a relatively moderate (16 weeks) exposure to a high-fat diet has the ability to increase brain oxidative stress altering Nrf2 signaling and impair cognitive function in aged animals [41].

Sulforaphane (SFN), a xenobiotic present in cruciferous vegetables such as broccoli, has been demonstrated to induce Nrf2-driven genes in rodent brains and microvasculature and by this to reduce brain damages in a traumatic brain injury model [42]. SFN administration initiated at $1 \mathrm{~h}$ post-cortical impact injury has been shown to improve cognitive function, in particular spatial learning and memory, and to reduce working memory dysfunction [43].

In a model of neonatal hypoxia-ischemia, pretreatment with SFN increased the expression of Nrf2 and HO-1 in the mouse brain and reduced infarct ratio [44]. Numerous other nonnutrients contained in food and plants have been ascribed to the list of Nrf2 activators, and among these several food-contained antioxidant polyphenols. One of the most important aspects of current polyphenol research is the focus on the neuroprotective capacity endowed by these molecules that seems to be due mostly to their ability to activate different defensive molecular pathways, instead to involve just their intrinsic antioxidant properties. In this regard, our and other laboratories have recently demonstrated the critical role of Nrf2/HO-1 activation by some of these neuroprotective compounds, providing insight into the possible therapeutic significance of a closely related group of polyphenols against neurodegenerative disorders and cognitive decline.

\section{Food Polyphenols and Neuroprotection}

In recent years, there has been a growing interest, supported by a large number of experimental and epidemiological studies, in the beneficial effects of some commonly used food-derived products in preventing various age-related pathologic conditions, including brain aging [45]. Spices and herbs often contain active phenolic substances endowed with potent antioxidative and chemopreventive properties [46] and a series of papers focused on specific neuroprotective effects of some of those polyphenols derived from nutritional sources $[47,48]$.

Although clinical studies on cognitive impairment and specific polyphenol supplementation have yet to be completed, a number of studies have indicated that inclusion of antioxidantrich foods in the diet can improve cognitive functioning in humans [49]. In elderly nondemented people, elevated dietary intake of flavonoid-rich foods was associated with better cognitive function [50]. Additionally, high flavonoid consumption was associated with attenuation of cognitive decline over a period of 10 years. Other studies have revealed that general dietary patterns, such as daily fruit and vegetable consumption, and adherence to a Mediterranean diet emphasizing vegetables and fruits can decrease the risk of developing dementia in aging humans $[51,52]$. Indeed, a large prospective study of the relationship between fruit and vegetable consumption (extending over a period of 10 to 16 years) indicated that vegetable intake is related to reduced cognitive decline [53]. This was 
particularly the case for green leafy vegetables and cruciferous vegetables. In addition, the protective effects of dietary polyphenols have also been reported for non-fruit and vegetable sources; for example, it has been shown that the intake of wine flavonoids is inversely related to the incidence of $\mathrm{AD}$ [54]. In general, polyphenols have the capacity to directly quench free radical species, and this characteristic certainly contributes to their neuroprotective effects. Beyond the antioxidant potential, most of these compounds appear to have a number of different molecular targets, impinging on several signaling pathways, involving NF- $\kappa B$, SIRT1, MAPK's, heat shock proteins (HSP), and showing pleiotropic activity on cells and tissues [55]. A possible general mechanism of polyphenols healing activity, correlated to their ability to overexpress highly protective inducible genes, is their involvement in the cellular stress response [56]. Most polyphenols show a bell-shaped doseresponse curve, presenting cellular toxicity at high dosage while inducing light chemical stress at lower concentration with activation of physiological hormesis in cells [57, 58], resulting in overexpression of defensive genes (such as those activated by Nrf2) and improved cellular survival. Our and other studies have demonstrated that curcumin, the pigment responsible for curry's characteristic yellow color, strongly induce HO-1 and other protein members of Phase II detoxification through the activation of Nrf2/ARE pathway in different tissues. Other plant-derived phenolic agents with analogous chemical structures to curcumin, such as carnosol [59], zerumbone [60], and rosolic acid [61], have been demonstrated to strongly activate HO-1 expression and to defend cells against oxidative stress. Furthermore, in our labs, we have demonstrated that other phenolics, such as caffeic acid phenethyl ester (CAPE), ethyl ferulate (EFE), and epigallocatechin-3-gallate (EGCG), are able to protect neurons via Nrf2/HO-1 induction (Fig. 1). Curcumin (1,7-bis[4hydroxy-3-methoxyphenyl]-1,6-hepta-diene-3,5-dione), a coloring agent and food additive commonly used in Indian culinary and traditional medical preparations from time immemorial, is extracted from the rhizome of Curcuma longa [62].

It is a polyphenolic substance that has the potential to inhibit lipid peroxidation and to effectively intercept and neutralize ROS (superoxide, peroxyl, hydroxyl radicals) [63, 64] and NO-based free radicals (nitric oxide and peroxynitrite) [65]. In this regard, curcumin has been demonstrated to be several times more potent than vitamin $\mathrm{E}$ [66]. The free radical chemistry of curcumin has focused on the redox peculiarities of its phenol ring, and the possible involvement of the $\beta$-diketone moiety in the antioxidant action of curcumin has been considered $[67,68]$. In a biological context, a poor correlation between in vitro and in vivo antioxidant properties of natural compounds has been observed [69]. Beyond its ROS quencher activity, curcumin effects have been mostly associated to its ability to interfere at molecular level with numerous cellular pathways. Of particular interest is the capacity of curcumin to inhibit COX-1 and COX-2 enzymes [70] and to reduce the activation of nuclear transcription factor NF-kB [71]. Its anti-inflammatory properties and cancer-preventive activities have been consistently assessed by using in vitro and in vivo models of tumor initiation and promotion [72]. In addition to its ability to scavenge carcinogenic free radicals [73], curcumin also interferes with cell growth through inhibition of protein kinases. Although the exact mechanisms by which curcumin promotes these effects remains to be elucidated, the electrophilic properties of this yellow pigment appear to be an essential component underlying its pleiotropic biological activities. Curcumin contains two 
electrophilic a, $\beta$-unsaturated carbonyl groups, which can react with nucleophiles such as glutathione [74]. By virtue of its Michael reaction acceptor function and its electrophilic characteristics, curcumin has been recently demonstrated to induce Phase II detox system $[75,76]$, mostly through the activation of Nrf2/ARE [77]. This finding is in agreement with other studies demonstrating that curcumin can increase the activity of $\boldsymbol{\gamma}$-glutamyl-cysteinyl synthetase and other GSH-linked detoxifying enzymes [78]. Recent and novel data from our group revealed that low concentrations of curcumin potently induces HO-1 expression and activity in vascular endothelial cells [79], in rat astrocytes [80], and in cultured hippocampal neurons [81]. In our experiments, we also demonstrated that preincubation (12 h) of cultured neurons with low concentration of curcumin resulted in an enhanced cellular resistance to glucose oxidase-mediated oxidative damage; this cytoprotective effect was considerably attenuated by zinc protoporphyrin IX, a specific inhibitor of HO activity. In other experiments, we demonstrated the efficacy of curcumin to protect cortical neurons against apoptotic cell death induced by $\beta$-amyloid peptide (1-40) [82]. The ability of curcumin to induce HO-1 can explain, at least in part, its strong antioxidant and anti-inflammatory properties, which depend more on its ability to activate cellular signals than on its radical scavenger effect [83]. A recent research demonstrated the ability of curcumin to reduce brain damages in a rat model of brain focal ischemia, via the induction of Nrf2/HO-1 [84]. The involvement of curcumin in restoring cellular homeostasis and rebalancing redox equilibrium by the activation of defensive genes suggests that it might be a useful adjunct also in brain aging and AD treatment [85]. Neuroprotective effects of curcumin have been demonstrated by Rajakrishnan and colleagues [86] in ethanol-induced brain damage, in which oral administration of curcumin to rats caused a significant reversal in lipid peroxidation, brain lipid modifications, as well as increase in glutathione levels. Epidemiological studies suggested that curcumin, as one of the most prevalent nutritional and medicinal compounds used by the Indian population, is responsible for the significantly reduced (4.4-fold) incidence of AD in India compared to USA [87]. Furthermore, elderly Singaporeans who ate curry with turmeric had higher MMSE scores than those who did not [88]. However, the relatively short duration of follow-up, cultural factors, and other potential confounders suggest caution in interpreting these findings. Consistent with these data, Cole and colleagues demonstrated in an Alzheimer transgenic mouse model ( $\mathrm{Tg} 2576)$ treated for 6 months that dietary curcumin resulted in a suppression of indices of inflammation and oxidative brain damage and reversed $A \beta$-induced cognitive deficits [89, 90]. Additional researches from the same group demonstrated that curcumin was a better A $\beta 40$ aggregation inhibitor than ibuprofen and naproxen and prevented $\mathrm{A} \beta 42$ oligomer formation and toxicity at very low concentration (between 0.1 and $1.0 \mu \mathrm{M}$ ) [91]. They also showed that curcumin readily entered the brain in vivo, thus inhibiting the formation of $A \beta$ oligomers and their toxicity. Among the several mechanisms by which curcumin is able to clear amyloid is the induction of HSPs that function as molecular chaperones to block protein aggregate formation [92]. Curcumin is also highly lipophilic and might cross the BBB and reach the brain. In fact, although its bioavailability is very low, since the drug is rapidly metabolized by conjugation, curcumin may reach brain concentrations sufficient to activate signal transduction events and to decrease A $\beta$ aggregation [93]. Recently, curcumin has been evaluated in a pilot clinical trial in AD patients, but preliminary results did not shown measurable beneficial effects [94]. Caffeic acid phenethyl ester is a phenolic structurally 
related to curcumin, originating from plants. CAPE is, in fact, an active component of propolis derived from the bark of conifer trees and carried by honeybees to their hives. The similarity to curcumin is striking because CAPE is also a Michael reaction acceptor that has a broad spectrum of biological activities, including anti-inflammatory [95, 96], antioxidant [97], and anticancer [98] effects. We have reported that CAPE is a potent inducer of HO-1 in astroglial cells and in neurons [80, 82]. CAPE is capable of transcriptionally activating a gene battery that also includes phase II detoxifying enzymes, through the ARE/Nrf2 pathway, demonstrating a neuroprotective capacity comparable to curcumin. Ferulic acid, which is the precursor of lignin biosynthesis, has long been recognized for its antioxidative and anti-inflammatory activities [99]. Cinnamic acid derivatives, including ferulic acid, are abundant in plants, playing important roles in the cross-linking of the cell walls of various lipids. Ferulic acid is found in many fruits and vegetables such as the tomato [100]. Tomato consumption has been demonstrated to result in absorption and excretion of ferulic acid by humans [101]. Ferulic acid has been demonstrated to have antioxidant activity against peroxynitrite [102] and against lipid peroxidation [103, 104]. It has been demonstrated that ferulic acid was protective against protein oxidation and lipid peroxidation in synaptosomal membranes and against cell death and protein carbonyl formation in neuronal cell culture induced by the peroxyl radical initiator AAPH [105]. Although it has been shown to be effective in in vitro experiments, its low lipophilicity impairs its in vivo efficiency, bioavailability, and stability. Ethyl ferulate, the naturally occurring ester derivative of ferulic acid, is present in various systems of many plants, such as the solanaceae family, as a trace constituent [106, 107]. In comparison with the corresponding acid form, EFE is more lipophilic and has been shown to possess better scavenging properties toward both hydroxyl radicals and superoxide anions. In a recent study of the inhibitory effects of antioxidants on lipid oxidation, EFE was shown to prevent autooxidation of model substrates by extending the induction time of this process [108].

Data from our lab clearly indicate that EFE is able, at low concentrations, to induce HO-1 protein expression and activity in cell lines of rat astrocytes and hippocampal neurons [109]. In our study, we also demonstrated the cytoprotective effects of EFE against oxidative damage in neuronal cells. Accordingly, other studies [110] have shown that EFE exerts strong neuroprotection against the oxidative stress and neurotoxicity induced by amyloid $\beta 1-42$. Green tea, one of the most widely consumed beverages, has recently attracted scientific attention as a potential nutritional strategy to prevent a broad range of age-related chronic disorders, including cardiovascular diseases [111, 112], cancer [113], obesity [114], diabetes [115], and neurodegenerative pathologies [116]. Moreover, a number of epidemiological studies have suggested that consuming green tea on a daily basis, as part of a lifestyle, might reduce the onset of age related diseases and improve longevity [117]. The health-promoting effects of green tea consumption are mainly attributed to its polyphenol content, which represents $35 \%$ of the dry weight. Compared with black tea, green tea is particularly rich in catechins that include: (-)-EGCG, (-)-epicatechin-3-gallate, (-)epigallocatechin, and epicatechin. EGCG is the most active and abundant compound in green tea, representing approximately $43 \%$ of the total phenols. Many of the aforementioned beneficial effects of green tea on age-related diseases have been linked to its EGCG content. EGCG possesses antioxidant and anti-inflammatory properties, which include the capacity 
to inhibit overexpression of cyclooxygenase- 2 and nitric oxide synthase [118]. It also induces apoptosis in several types of cancer cells by inactivating some transcription factors, such as NF- $\mathrm{kB}, \mathrm{AP}-1$, and STAT-1 [119]. EGCG prevents cancer cell invasion, angiogenesis, and metastasis by downregulating the expression of matrix metalloproteinases and by inhibiting the cell adhesion function [120]. Several reports have also shown the ability of EGCG to induce a general xenobiotic response in the target cells, activating multiple defense genes. In particular, some studies have recently demonstrated that the neuroprotective mechanisms of EGCG are partly due to increasing activities of antioxidant enzymes and decreasing advanced glycation end-product-induced damage in rat brain or neuronal cells [121]. In a recent study, we attempted to determine the molecular mechanisms underlying the antioxidant effects of EGCG in neurons exposed to oxidative stress, by focusing on the ability of this compound to upregulate HO-1 expression and other adaptive enzymes involved in cellular stress response. In accordance with other studies [122], we demonstrated the ability of EGCG to activate HO-1 by the ARE/Nrf2 pathway and by the induction of HO-1 to protect neurons against oxidative damage [123]. A recent study has demonstrated the ability of tea epicatechins to protect neurons and reduce brain infarct size of mice subjected to middle cerebral artery occlusion or to $\mathrm{N}$-methyl-D-aspar-tateinduced excitotoxicity. Neuroprotection was mostly abolished in mice lacking $\mathrm{HO} 1$ or the transcriptional factor Nrf2 and in neurons derived from these knockout mice [124].

\section{Conclusion}

Research described above suggests that polyphenolic compounds exhibit potent antioxidant and anti-inflammatory activities that may reduce neurodegeneration. The majority of in vitro and in vivo studies conducted so far have attributed the protective effect of bioactive polyphenols to their chemical reactivity toward free radicals and their capacity to prevent the oxidation of important intracellular components. However, observations from our and other laboratories reveal a potential novel aspect in the mode of action of curcumin and curcuminlike compounds; the ability to activate a physiological hormetic response in the cells, and the ultimate stimulation of the Nrf2 pathway, with the expression of phase II and HO-1 genes, is likely to account for the established and powerful antioxidant/anti-inflammatory properties of these plant-derived compounds. Because the HO-1 gene can be stimulated at transcriptional levels by a plethora of noxious stimuli, the use of plant-derived natural substances to trigger HO-1 expression and other intra-cellular defensive systems via Nrf2 activation, would clearly offer a greater advantage for therapeutic and preventive purposes. Recent evidences suggest that that oxidative stress and inflammation can interfere with the physiology of learning and memory, and the above-mentioned pathways might play a relevant role in the pathogenic mechanism underlying many major cognitive and behavioral disorders. Many of the polyphenols able to activate Nrf2 have been shown also to modulate levels and activity of norepinephrine, serotonin, dopamine, and glutamate, the principal transmitters involved in addiction. As addiction may be a form of pathological learning, the role played by food polyphenols may be hypothesized. It needs to be emphasized that curcumin and other plant constituents eventually could become part of the human diet and consumed daily as supplements. A number of studies identified a novel class of natural substances that could be used for therapeutic purposes as potent activators of $\mathrm{Nrf} 2$ in the 
protection of tissues against inflammatory and neurodegenerative conditions. Further in vitro and in vivo studies using curcumin-like molecules will give important information on the feasibility of developing new pharmacological strategies for maximizing Nrf2 activity in targeted tissues as an alternative to or in combination with Nrf2 or HO-1 gene therapy.

\section{References}

1. Alzheimer's Association. Alzheimer's disease facts and figures. Alzheimers Dement. 2009; 5:234270. [PubMed: 19426951]

2. Rojo LE, et al. Neuroinflammation: implications for the pathogenesis and molecular diagnosis of Alzheimer's disease. Arch Med Res. 2008; 39:1-16. [PubMed: 18067990]

3. Butterfield DA, et al. Evidence of oxidative damage in Alzheimer's disease brain: central role for amyloid beta-peptide. Trends Mol Med. 2001; 7:548-554. [PubMed: 11733217]

4. Halliwell B. Biochemistry of oxidative stress. Biochem Soc Trans. 2007; 35:1147-1150. [PubMed: 17956298]

5. Butterfield DA, Sultana R. Redox proteomics identification of oxidatively modified brain proteins in Alzheimer's disease and mild cognitive impairment: insights into the progression of this dementing disorder. J Alzheimers Dis. 2007; 12:61-72. [PubMed: 17851195]

6. Katzman R, Saitoh T. Advances in Alzheimer's disease. FASEB J. 1991; 5:278-286. [PubMed: 2001787]

7. Finkel T, Holbrook NJ. Oxidants, oxidative stress and the biology of aging. Nature. 2000; 408:239247. [PubMed: 11089981]

8. Calabrese V, et al. Nitric oxide and cellular stress response in brain aging and neurodegenerative disorders: the role of vitagenes. In Vivo. 2004; 18:245-267. [PubMed: 15341181]

9. Skovronsky DM, Lee VM, Trojanowski JQ. Neurodegenerative diseases: new concepts of pathogenesis and their therapeutic implications. Annu Rev Pathol. 2006; 1:151-170. [PubMed: 18039111]

10. Racchi M, et al. Alzheimer's disease; new diagnostic and therapeutic tools. Immun Ageing. 2008; 5:7. [PubMed: 18700965]

11. Vatassery GT, Fahn S, Kuskowski MA. The Parkinson Study Group. Alpha tocopherol in CSF of subjects taking high dose vitamin E in the DATATOP study. Neurology. 1998; 50:1900-1902. [PubMed: 9633757]

12. Johnson JA, et al. The Nrf2-ARE pathway: An indicator and modulator of oxidative stress in neurodegeneration. Ann NY Acad Sci. 2008; 1147:61-69. [PubMed: 19076431]

13. Innamorato NG, et al. The transcription factor Nrf2 is a therapeutic target against brain inflammation. J Immunol. 2008; 181(1):680-689. [PubMed: 18566435]

14. Sun Z, et al. Keap1 controls postinduction repression of the Nrf2-mediated antioxidant response by escorting nuclear export of Nrf2. Mol Cell Biol. 2007; 27:6334-6349. [PubMed: 17636022]

15. Yamamoto T, et al. Physiological significance of reactive cysteine residues of Keap1 in determining Nrf2 activity. Mol Cell Biol. 2008; 28:2758-2770. [PubMed: 18268004]

16. Malhotra D, et al. Global mapping of binding sites for Nrf2 identifies novel targets in cell survival response through ChIP-Seq profiling and network analysis. Nucleic Acids Res. 2010; 38(17): 5718-5734. [PubMed: 20460467]

17. Jakel RJ, et al. Nrf2-mediated protection against 6-hydroxydopamine. Brain Res. 2007; 1144:192201. [PubMed: 17336276]

18. Innamorato NG, et al. Different susceptibility to the Parkinson's toxin MPTP in mice lacking the redox master regulator Nrf2 or its target gene heme oxygenase-1. PLoS ONE. 2010; 5(7):e11838. [PubMed: 20676377]

19. Rojo AI, et al. Nrf2 regulates microglial dynamics and neuroinflammation in experimental Parkinson's disease. Glia. 2010; 58(5):588-598. [PubMed: 19908287] 
20. Vargas MR, et al. Increased glutathione biosynthesis by Nrf2 activation in astrocytes prevents p75NTR-dependent motor neuron apoptosis. J Neurochem. 2006; 97(3):687-696. [PubMed: 16524372]

21. Vargas MR, et al. Nrf2 activation in astrocytes protects against neurodegeneration in mouse models of familial amyo-trophic lateral sclerosis. J Neurosci. 2008; 28(50):13574-13581. [PubMed: 19074031]

22. Ramsey CP, et al. Expression of Nrf2 in neurodegenerative diseases. J Neuropathol Exp Neurol. 2007; 66:75-85. [PubMed: 17204939]

23. Kanninen K, et al. Nuclear factor erythroid 2-related factor 2 protects against beta amyloid. Mol Cell Neurosci. 2008; 39:302-313. [PubMed: 18706502]

24. Wruck CJ, et al. Kavalactones protect neural cells against amyloid beta peptideinduced neurotoxicity via extracellular signal-regulated kinase 1/2-dependent nuclear factor erythroid 2related factor 2 activation. Mol Pharmacol. 2008; 73:1785-1795. [PubMed: 18334601]

25. Kanninen K, et al. Intrahippocampal injection of a lentiviral vector expressing Nrf2 improves spatial learning in a mouse model of Alzheimer's disease. Proc Natl Acad Sci USA. 2009; 106:16505-16510. [PubMed: 19805328]

26. Chen PC, et al. Nrf2-mediated neuroprotection in the MPTP mouse model of Parkinson's disease: Critical role for the astrocyte. Proc Natl Acad Sci USA. 2009; 106:2933-2938. [PubMed: 19196989]

27. Abraham NG, Kappas A. Pharmacological and clinical aspects of heme oxygenase. Pharmacol Rev. 2008; 60(1):79-127. [PubMed: 18323402]

28. Takahashi M, et al. Amyloid precursor proteins inhibit heme oxygenase activity and augment neurotoxicity in Alzheimer's disease. Neuron. 2002; 28:461-473.

29. Schipper HM. Heme oxygenase-1: role in brain aging and neurodegeneration. Exp Gerontol. 2000; 35:821-830. [PubMed: 11053673]

30. Colombrita $\mathrm{C}$, et al. Regional rat brain distribution of heme oxygenase-1 and manganese superoxide dismutase mRNA: relevance of redox homeostasis in the aging processes. Exp Biol Med. 2003; 228:517-524.

31. Chen K, Gunter K, Maines MD. Neurons overexpressing heme oxygenase-1 resist oxidative stressmediated cell death. J Neurochem. 2000; 75:304-312. [PubMed: 10854275]

32. Le WD, Xie WJ, Appel SH. Protective role of heme oxygenase-1 in oxidative stress-induced neuronal injury. J Neurosci Res. 1999; 56:652-658. [PubMed: 10374820]

33. Willis D, et al. Heme oxygenase: a novel target for the modulation of the inflammatory response. Nat Med. 1996; 2:87-90. [PubMed: 8564848]

34. Poss KD, Tonegawa S. Reduced stress defense in heme oxygenase 1-deficient cells. Proc Natl Acad Sci USA. 1997; 94:10925-10930. [PubMed: 9380736]

35. Yachie A, et al. Oxidative stress causes enhanced endothelial cell injury in human heme oxygenase-1 defi- ciency. J Clin Invest. 1999; 103:129-135. [PubMed: 9884342]

36. Paine A, et al. Signaling to heme oxygenase-1 and its anti-inflammatory therapeutic potential. Biochem Pharmacol. 2010; 80(12):1895-1903. [PubMed: 20643109]

37. Pamplona A, et al. Heme oxygenase-1 and carbon monoxide suppress the pathogenesis of experimental cerebral malaria. Nat Med. 2007; 13(6):703-710. [PubMed: 17496899]

38. Chora AA, et al. Heme oxygenase-1 and carbon monoxide suppress autoimmune neuroinflammation. J Clin Invest. 2007; 117(2):438-47. [PubMed: 17256058]

39. Dumont M, et al. Triterpenoid CDDOmethylamide improves memory and decreases amyloid plaques in a transgenic mouse model of Alzheimer's disease. J Neurochem. 2009; 109(2):502-512. [PubMed: 19200343]

40. Son TG, et al. Plumbagin, a novel Nrf2/ARE activator, protects against cerebral ischemia. J Neurochem. 2010; 112(5):1316-1326. [PubMed: 20028456]

41. Morrison CD, et al. High fat diet increases hippocampal oxidative stress and cognitive impairment in aged mice: implications for decreased Nrf2 signaling. J Neurochem. 2010; 114(6):1581-1589. [PubMed: 20557430] 
42. Zhao J, et al. Enhancing expression of Nrf2-driven genes protects the blood brain barrier after brain injury. J Neurosci. 2007; 27:10240-10248. [PubMed: 17881530]

43. Dash P, et al. Sulforaphane improves cognitive function administered following traumatic brain injury. Neurosci Lett. 2009; 460(2):103-107. [PubMed: 19515491]

44. Ping Z, et al. Sulforaphane protects brains against hypoxic-ischemic injury <!- through induction of Nrf2-dependent phase 2 enzyme. Brain Res. 2010; 1343:178-185. [PubMed: 20417626]

45. Gomez-Pinilla F. Brain foods: the effects of nutrients on brain function. Nat Rev Neurosci. 2008; 9:568-578. [PubMed: 18568016]

46. Nakatani N. Phenolic antioxidants from herbs and spices. Biofactors. 2000; 13:141-146. [PubMed: 11237173]

47. Butterfield D, et al. Nutritional approaches to combat oxidative stress in Alzheimer's disease. J Nutr Biochem. 2002; 13:444-461. [PubMed: 12165357]

48. Sun AY, et al. Botanical phenolics and brain health. Neuromolecular Med. 2008; 10:259-274. [PubMed: 19191039]

49. Van Dyk K, Sano M. The impact of nutrition on cognition in the elderly. Neurochem Res. 2007; 32:893-904. [PubMed: 17342414]

50. Letenneur L, et al. Flavonoid intake and cognitive decline over a 10-year period. Am J Epidemiol. 2007; 165:1364-1371. [PubMed: 17369607]

51. Barberger-Gateau P, et al. Dietary patterns and risk of dementia: the Three-City cohort study. Neurology. 2007; 69:921-930.

52. Scarmeas N, et al. Mediterranean diet, alzheimer disease, and vascular mediation. Arch Neurol. 2006; 63:1709-1717. [PubMed: 17030648]

53. Kang JH, Ascherio A, Grodstein F. Fruit and vegetable consumption andcognitive decline in aging women. Ann Neurol. 2005; 57:713-720. [PubMed: 15852398]

54. Commenges D, et al. Intake of flavonoids and risk of dementia. Eur J Epidemiol. 2000; 16:357363. [PubMed: 10959944]

55. Kim J, Lee HJ, Lee KW. Naturally occurring phytochem-icals for the prevention of Alzheimer's disease. J Neurochem. 2010; 112(6):1415-30. [PubMed: 20050972]

56. Mattson MP, Son TG, Camandola S. Viewpoint: mechanisms of action and therapeutic potential of neurohormetic phytochemicals. Dose Response. 2007; 5(3):174-186. [PubMed: 18648607]

57. Calabrese EJ. Neuroscience and hormesis: overview and general findings. Crit Rev Toxicol. 2008; 38(4):249-52. [PubMed: 18432418]

58. Rattan SI, Fernandes RA, Demirovic D, Dymek B, Lima CF. Heat stress and hormetin-induced hormesis in human cells: effects on aging, wound healing, angiogenesis, and differentiation. Dose Response. 2009; 7(1):90-103. [PubMed: 19343114]

59. Martin D, et al. Regulation of heme oxygenase-1 expression through the phosphatidylinositol 3kinase/Akt pathway and the Nrf2 transcription factor in response to the antioxidant phytochem-ical carnosol. J Biol Chem. 2004; 279:8919-8929. [PubMed: 14688281]

60. Surh YJ, Kundu JK, Na HK. Nrf2 as a master redox switch in turning on the cellular signaling involved in the induction of cytoprotective genes by some chemopreventive phytochemicals. Planta Med. 2008; 74(13):1526-1539. [PubMed: 18937164]

61. Foresti R, et al. Differential activation of heme oxygenase-1 by chalcones and rosolic acid in endothelial cells. J Pharmacol Exp Ther. 2005; 312:686-693. [PubMed: 15537827]

62. Ammon HPT, Wahl MA. Pharmacology of Curcuma Longa. Planta Med. 1991; 57:1-7. [PubMed: 2062949]

63. Priyadarsini KI, Guha SN, Rao MN. Physicochemical properties and antioxidant activities of methoxy phenols. Free Radic Biol Med. 1998; 24:933-941. [PubMed: 9607603]

64. Martin-Aragon S, Benedi JM, Villar AM. Modifications on antioxidant capacity and lipid peroxidation in mice under fraxetin treatment. J Pharm Pharmacol. 1997; 49:49-52. [PubMed: 9120770]

65. Sreejayan A, Rao MN. Nitric oxide scavenging by curcuminoids. J Pharm Pharmacol. 1997; 49:105-107. [PubMed: 9120760] 
66. Zhao BL, et al. Scavenging effect of extracts of green tea and natural antioxidants on active oxygen radicals. Cell Biophys. 1989; 14:175-185. [PubMed: 2472207]

67. Masuda T, et al. Chemical studies on antioxidant mechanism of curcuminoid: analysis of radical reaction products from curcumin. J Agric Food Chem. 1999; 47:71-77. [PubMed: 10563852]

68. Jovanovic SV, et al. How curcumin works preferentially with soluble antioxidants. J Am Chem Soc. 2001; 123:3064-3068. [PubMed: 11457017]

69. Pun PB, et al. Ageing in nematodes: do antioxidants extend lifespan in Caenorhabditis elegans? Biogerontology. 2010; 11:17-30. [PubMed: 19350411]

70. Ramos-Gomez M, et al. Sensitivity to carcinogenesis is increased and chemoprotective efficacy of enzyme inducers is lost in nrf2 transcription factor-deficient mice. Proc Natl Acad Sci USA. 2001; 98:3410-3415. [PubMed: 11248092]

71. Singh S, Aggarwal BB. Activation of transcription factor NF- $\kappa \mathrm{B}$ is suppressed by curcumin (diferuloylmethane). J Biol Chem. 1995; 270:24995-25000. [PubMed: 7559628]

72. Huang MT, Newmark HL, Frenkel K. Inhibitory effects of curcumin on tumorigenesis in mice. J Cell Biochem Suppl. 1997; 27:26-34. [PubMed: 9591190]

73. Abe Y, Hashimoto S, Horie T. Curcumin inhibition of inflammatory cytokine production by human peripheral blood monocytes and alveolar macrophages. Pharmacol Res. 1999; 39:41-47. [PubMed: 10051376]

74. Awasthi S, et al. Curcumin-glutathione interactions and the role of human glutathione S-transferase P1-1. Chem Biol Interact. 2000; 128:19-38. [PubMed: 10996298]

75. Dinkova-Kostova AT, Talalay P. Relation of structure of curcumin analogs to their potencies as inducers of Phase 2 detoxification enzymes. Carcinogenesis. 1999; 20:911-914. [PubMed: 10334211]

76. Dinkova-Kostova AT, et al. Potency of Michael reaction acceptors as inducers of enzymes that protect against carcino-genesis depends on their reactivity with sulfhydryl groups. Proc Natl Acad Sci USA. 2001; 98:3404-3409. [PubMed: 11248091]

77. Balogun E, et al. Curcumin activates the haem oxygenase-1 gene via regulation of Nrf2 and the antioxidant-responsive element. Biochem J. 2003; 371:887-895. [PubMed: 12570874]

78. Singhal SS, et al. The effect of curcumin on glutathione-linked enzymes in K562 human leukemia cells. Toxicol Lett. 1999; 109:87-95. [PubMed: 10514034]

79. Motterlini R, et al. Curcumin, an antioxidant and anti-inflammatory agent, induces heme oxygenase-1 and protects endothelial cells against oxidative stress. Free Radic Biol Med. 2000; 28:1303-1312. [PubMed: 10889462]

80. Scapagnini G, et al. Caffeic acid phenethyl ester and curcumin: a novel class of heme oxygenase-1 inducers. Mol Pharmacol. 2002; 61:554-561. [PubMed: 11854435]

81. Scapagnini G, et al. Curcumin activates defensive genes and protects neurons against oxidative stress. Antioxid Redox Signal. 2006; 8:395-403. [PubMed: 16677086]

82. Scapagnini, G., et al. Use of curcumin derivatives or CAPE in the manufacture of a medicament for the treatment of neuroprotective disorders. World Patent Number: WO 2004/075883 A1. 2004.

83. Goel A, Kunnumakkara AB, Aggarwal BB. Curcumin as - Curecuminll: from kitchen to clinic. Biochem Pharmacol. 2008; 75:787-809. [PubMed: 17900536]

84. Yang C, Zhang X, Fan H, Liu Y. Curcumin upregulates transcription factor Nrf2, HO-1 expression and protects rat brains against focal ischemia. Brain Res. 2009; 1282:133-41. [PubMed: 19445907]

85. Sikora E, Scapagnini G, Barbagallo M. Curcumin, inflammation, ageing and age-related diseases. Immun Ageing. 2010; 7(1):1. [PubMed: 20205886]

86. Rajakrishnan V, et al. Neuroprotective role of curcumin from curcuma longa on ethanol induced brain damage. Phytother Res. 1999; 13:571-574. [PubMed: 10548748]

87. Chandra V, et al. Incidence of Alzheimer's disease in a rural community in India: the Indo-US study. Neurology. 2001; 57:985-989. [PubMed: 11571321]

88. Ng TP, et al. Curry consumption and cognitive function in the elderly. Am J Epidemiol. 2006; 164:898-906. [PubMed: 16870699] 
89. Lim GP, et al. The curry spice curcumin reduces oxidative damage and amyloid pathology in an Alzheimer transgenic mouse. J Neurosci. 2001; 21:8370-8377. [PubMed: 11606625]

90. Frautschy SA, et al. Phenolic anti-inflammatory antioxidant reversal of Abeta induced cognitive deficits and neuropathology. Neurobiol Aging. 2001; 22:993-1005. [PubMed: 11755008]

91. Yang F, et al. Curcumin inhibits formation of amyloid beta oligomers and fibrils, binds plaques and reduces amyloid in vivo. J Biol Chem. 2005; 280:5892-5901. [PubMed: 15590663]

92. Cole GM, Teter B, Frautschy SA. Neuroprotective effects of curcumin. Adv Exp Med Biol. 2007; 595:197-212. [PubMed: 17569212]

93. Begum AN, et al. Curcumin structure-function, bioavail-ability and efficacy in models of neuroinflammation and Alzheimer's disease. J Pharmacol Exp Ther. 2008; 326:196-208. [PubMed: 18417733]

94. Baum L, et al. Six-month randomized, placebo-controlled, double-blind, pilot clinical trial of curcumin in patients with Alzheimer disease. J Clin Psychopharmacol. 2008; 28:110-113. [PubMed: 18204357]

95. Michaluart $P$, et al. Inhibitory effects of caffeic acid phenethyl ester on the activity and expression of cyclooxygenase- 2 in human oral epithelial cells and in a rat model of inflammation. Cancer Res. 1999; 59:2347-2352. [PubMed: 10344742]

96. Natarajan K, et al. Caffeic acid phenethyl ester is a potent and specific inhibitor of activation of nuclear transcription factor NF-kappa B. Proc Natl Acad Sci USA. 1996; 93:9090-9095. [PubMed: 8799159]

97. Chen YJ, Shiao MS, Wang SY. The antioxidant caffeic acid phenethyl ester induces apoptosis associated with selective scavenging of hydrogen peroxide in human leukemic HL-60 cells. Anticancer Drugs. 2001; 12:143-149. [PubMed: 11261888]

98. Huang MT, et al. Inhibitory effects of caffeic acid phenethyl ester (CAPE) on 12-Otetradecanoylphorbol-13-acetate-induced tumor promotion in mouse skin and the synthesis of DNA, RNA and protein in HeLa cells. Carcinogenesis. 1996; 17:761-765. [PubMed: 8625488]

99. Graf E. Antioxidant potential of ferulic acid. Free Radic Biol Med. 1992; 13:435-448. [PubMed: 1398220]

100. Qureshi MJ, Blain JA. Antioxidant activity in tomato extracts. Nucleus Karachi. 1976; 13:29-33.

101. Bourne LC, Rice-Evans C. Biovailability of ferulic acid. Biochem Biophys Res Commun. 1998; 253:222-227. [PubMed: 9878519]

102. Pannala R, et al. Inhibition of peroxynitrite dependent tyrosine nitration by hydroxycinnamates: nitration or electron donation. Free Radic Biol Med. 1998; 24:594-606. [PubMed: 9559872]

103. Castelluccio C, et al. Antioxidant potential of intermediates in phenylpropanoid metabolism in higher plants. FEBS Lett. 1995; 368:188-192. [PubMed: 7615079]

104. Bourne L, Rice-Evans C. The effect of the phenolic antioxidant ferulic acid on the oxidation of low density lipoprotein depends on the pro-oxidant used. Free Radic Res. 1997; 27:337-344. [PubMed: 9350437]

105. Kanski J, et al. Ferulic acid antioxidant protection against hydroxyl and peroxyl radical oxidation in synaptosomal and neuronal cell culture systems in vitro: structure-activity studies. J Nutr Biochem. 2002; 13:273-281. [PubMed: 12015157]

106. Clifford MN. Chlorogenic acids and other cinnamates — nature, occurrence and dietary burden. J Sci Food Agric. 1999; 79:362-372.

107. Kroon PA, Williamson G. Hydroxycinnamates in plants and food: current and future perspectives. J Sci Food Agric. 1999; 79:355-361.

108. Kikuzaki H, et al. Antioxidant properties of ferulic acid and its related compounds. J Agric Food Chem. 2002; 50:2161-2168. [PubMed: 11902973]

109. Scapagnini G, et al. Ethyl ferulate, a lipophilic polyphenol, induces HO-1 and protects rat neurons against oxidative stress. Antioxid Redox Signal. 2004; 6:811-818. [PubMed: 15345140]

110. Perluigi M, et al. In vivo protective effects of ferulic acid ethyl ester against amyloid-beta peptide 1-42-induced oxidative stress. J Neurosci Res. 2009; 84:418-26.

111. Sano J, et al. Effects of green tea intake on the development of coronary artery disease. Circ J. 2004; 68:665-670. [PubMed: 15226633] 
112. Wolfram S. Effects of green tea and EGCG on cardiovascular and metabolic health. J Am Coll Nutr. 2007; 26:373-388.

113. Moyers SB, Kumar NB. Green tea polyphenols and cancer chemoprevention: multiple mechanisms and endpoints for phase II trials. Nutr Rev. 2004; 62:204-211. [PubMed: 15212320]

114. Boschmann M, Thielecke F. The effects of epigallocatechin-3-gallate on thermogenesis and fat oxidation in obese men: a pilot study. J Am Coll Nutr. 2007; 26:389-395.

115. Potenza MA, et al. Epigallocatechin gallate, a green tea polyphenol, improves endothelial function and insulin sensitivity, reduces bood pressure and protects against myocardial ischemia/ reperfusion injury in spontaneously hypertensive rats (SHR). Am J Physiol Endocrinol Metab. 2007; 292:1378-1387.

116. Mandel S, et al. Cell signaling pathways in the neuro-protective actions of the green tea polyphenol (-)-epigallocatechin-3-gallate: implications for neurodegenerative diseases. J Neurochem. 2004; 88:1555-1569. [PubMed: 15009657]

117. Khan N, Mukhtar H. Tea polyphenols for health promotion. Life Sci. 2007; 81:519-533. [PubMed: 17655876]

118. Ahmed S, et al. Green tea polyphenol epigallocatechin-3-gallate inhibits the IL-1 beta-induced activity and expression of cyclooxygenase- 2 and nitric oxide synthase- 2 in human chon-drocytes. Free Radic Biol Med. 2002; 33:1097-1105. [PubMed: 12374621]

119. Kim SJ, et al. Epigallocatechin-3-gallate suppresses NF-kappaB activation and phosphorylation of p38 MAPK and JNK in human astrocytoma U373MG cells. J Nutr Biochem. 2007; 18:587-596. [PubMed: 17446059]

120. Khan N, Mukhtar H. Multitargeted therapy of cancer by green tea polyphenols. Cancer Lett. 2008; 269:269-280. [PubMed: 18501505]

121. Srividhya R, et al. Attenuation of senescence-induced oxidative exacerbations in aged rat brain by (-)-epigallocatechin-3-gallate. Int J Dev Neurosci. 2008; 26:217-223. [PubMed: 18207349]

122. Kweon MH, et al. Constitutive overexpression of Nrf2-dependent heme oxygenase-1 in A549 cells contributes to resistance to apoptosis induced by epigallocatechin 3-gallate. J Biol Chem. 2006; 281:33761-33772. [PubMed: 16950787]

123. Romeo L, et al. The major green tea polyphenol, (-)-epigallocatechin-3-gallate, induces heme oxygenase in rat neurons and acts as an effective neuroprotective agent against oxidative stress. J Am Coll Nutr. 2009; 28:492S-499S. [PubMed: 20234037]

124. Shah ZA, Li RC, Ahmad AS, Kensler TW, Yamamoto M, Biswal S, Doré S. The flavanol (-)epicatechin prevents stroke damage through the Nrf2/HO1 pathway. J Cereb Blood Flow Metab. 2010; 30(12):1951-1961. [PubMed: 20442725] 
<smiles>COc1cc(/C=C/C(=O)CC(=O)/C=C/c2ccc(O)c(OC)c2)ccc1O</smiles>

b<smiles>O=C(/C=C/c1ccc(O)c(O)c1)OCCc1ccccc1</smiles>

C<smiles>CCOC(=O)/C=C/c1ccc(O)c(OC)c1</smiles><smiles>O=C(OC1Cc2c(O)cc(O)cc2OC1c1cc(O)c(O)c(O)c1)c1cc(O)c(O)c(O)c1</smiles>

Fig. 1.

The chemical structures of curcumin (a), CAPE (b), EFE (c), (-)-EGCG (d) 